\title{
Cytotoxic effects of solvent-extracted active components of Salvia miltiorrhiza Bunge on human cancer cell lines
}

\author{
BOKYUNG SUNG ${ }^{1}$, HYE SUN CHUNG ${ }^{1}$, MINJUNG KIM ${ }^{1}$, YONG JUNG KANG ${ }^{1}$, DONG HWAN KIM ${ }^{1}$, \\ SEONG YEON HWANG ${ }^{1}$, MIN JO KIM ${ }^{1}$, CHEOL MIN KIM $^{2}$, HAE YOUNG CHUNG $^{1}$ and NAM DEUK KIM ${ }^{1}$ \\ ${ }^{1}$ Department of Pharmacy, College of Pharmacy, Pusan National University, Busan 609-735; \\ ${ }^{2}$ Department of Biochemistry, School of Medicine, Pusan National University, Yangsan 626-770, Republic of Korea
}

Received November 2, 2014; Accepted January 28, 2015

DOI: $10.3892 / \mathrm{etm} .2015 .2252$

\begin{abstract}
Herbal extracts and dietary supplements may be extracted from the medicinal plants used in traditional Chinese medicine, and are used increasingly commonly worldwide for their benefits to health and quality of life. Thus, ensuring that they are safe for human consumption is a critical issue for the preparation of plant extracts as dietary supplements. The present study investigated extracts of Salvia miltiorrhiza Bunge (S. miltiorrhiza), traditionally used in Asian countries to treat a variety of conditions, as a dietary supplement or as an ingredient in functional foods. Dried S. miltiorrhiza root was extracted with various solvents and under varying extraction conditions, and the effects of the extracts on the viability of five human cancer cell lines were compared. Extracts obtained using $100 \%$ ethanol and $100 \%$ acetone as solvents exhibited more potent effects compared with extracts obtained using 70 and $30 \%$ aqueous ethanol. Furthermore, the active components of $S$. miltiorrhiza ethanol extracts, known as tanshinones, were investigated. Dihydrotanshinone I was observed to exhibit a higher cytotoxic potential compared with the other tanshinones in the majority of the examined cell lines. Conversely, cryptotanshinone exhibited weak anti-cancer activity. In summary, the results of the present study suggest that the active components obtained from an ethanol extract of S. miltiorrhiza possess the potential to be used as ingredients in functional and health care foods that may be used to improve the effectiveness of chemotherapeutics in the prevention and/or treatment of cancer.
\end{abstract}

Correspondence to: Dr Nam Deuk Kim, Department of Pharmacy, College of Pharmacy, Pusan National University, 63 Beon-gil 2, Busan 609-735, Republic of Korea

E-mail: nadkim@pusan.ac.kr

Key words: Salvia miltiorrhiza Bunge, extraction, tanshinones, cancer

\section{Introduction}

The dried root of Salvia miltiorrhiza Bunge (S. miltiorrhiza; Radix Salvia Miltiorrhizae), known as Danshen in Chinese, Dansam in Korean and Tansen in Japanese, is one of the most commonly used substances in traditional Chinese medicine (TCM), and is also used in other Asian countries, including Korea and Japan (1). S. miltiorrhiza has been used in the treatment of a variety of conditions, including cardiovascular disease, cerebrovascular disease, diabetic vascular complications (1), liver dysfunction (2) and renal disease (3). Furthermore, Wen et al previously demonstrated that S. miltiorrhiza is effective in the treatment of gastrointestinal inflammatory disease (4). The active chemical constituents of S. miltiorrhiza root have been studied extensively. These active components, the majority of which have been identified and purified, are generally divided into two major groups; water-soluble phenolic compounds and lipophilic diterpene quinones (5). The lipid-soluble compounds, usually extracted using alcohol solvents, are rich in abietanoids and diterpene quinones (tanshinones). Numerous diterpenoid tanshinones have been extracted from $S$. miltiorrhiza, including dihydrotanshinone I, cryptotanshinone and tanshinones I and IIA, which are the three most commonly studied. These tanshinones have been reported to exhibit antioxidative (6-9), anti-inflammatory $(6,10,11)$, anti-allergic (12) and anti-cancer effects $(13,14)$.

In China, $S$. miltiorrhiza has been used as a medicine or dietary supplement for improving health. The oral administration of this medicinal herb appears to exhibit negligible effects on the pharmacokinetics of therapeutic agents, such as docetaxel or clopidogrel, indicating the potential benefits of combining S. miltiorrhiza with standard therapeutics (15). Furthermore, the combination of S. miltiorrhiza and its active constituents with other TCM or chemotherapeutic substances has been observed to result in more notable anti-cancer effects compared with either agent alone $(16,17)$. However, the safety of orally administered S. miltiorrhiza as a nutritional or dietary supplement is not known and may be a potential disadvantage to its use as a therapeutic.

The first step in the utilization of medicinal plants as dietary supplements, health foods/nutritional supplements or pharmaceuticals is the extraction of the bioactive constituents from 
the plant materials. The active components of plant materials are commonly extracted using a solvent. Numerous solvents, including methanol, ethanol, acetone and ethyl acetate, have previously been used to prepare extracts from plant materials, either alone or in combination and at varying aqueous dilutions. Ethanol is considered to be a particularly effective solvent, and its use is permitted by the food industry for the preparation of dietary supplements or functional foods, as it is safe for human consumption (18). Numerous S. miltiorrhiza preparations are currently available, including lipophilic and hydrophilic extracts. The ethanol extract of $S$. miltiorrhiza, which is rich in lipophilic constituents, is the most commonly used in Chinese clinics (19).

The present study aimed to compare the effects of a number of ethanol extracts of S. miltiorrhiza, obtained using ethanol at various aqueous dilutions as a solvent, with an extract obtained using acetone, one of the most common solvents for hydrophobic compounds, on the viability of cancer cells. In addition, the cytotoxic effects of the bioactive constituents indicated to be present in the most active fraction of $S$. miltiorrhiza were assessed in five human cancer cell lines.

\section{Materials and methods}

Reagents. Dihydrotanshinone I, cryptotanshinone and tanshinone I were purchased from Sigma-Aldrich (St. Louis, MO, USA). These reference tanshinones were dissolved in dimethylsulfoxide (DMSO) at a $100 \mu \mathrm{M}$ concentration and stored at $-20^{\circ} \mathrm{C}$ prior to the experiments, and further dilutions were performed in the culture medium. MTT was obtained from Amresco LLC (Solon, OH, USA) and RPMI-1640 medium, Dulbecco's modified Eagle's medium (DMEM), fetal bovine serum (FBS) and penicillin-streptomycin were purchased from HyClone (GE Healthcare, Logan, UT, USA).

Preparation of crude S. miltiorrhiza fractions. Extraction was conducted at room temperature by placing $50 \mathrm{~g}$ powdered S. miltiorrhiza root (Hangzhou Botanical Technology Co., Ltd., Hangzhou, China) in a 500-ml conical flask and adding $250 \mathrm{ml}$ acetone or 100,70 or $30 \%$ aqueous ethanol (v/v). In addition, an extraction using $30 \%$ aqueous ethanol at $4^{\circ} \mathrm{C}$ was conducted. The mouth of the conical flask was covered with aluminum foil and the contents left for $4 \mathrm{~h}$, allowing extraction of the active components into the solvent. Next, the extracts were separated from the residues by filtering the extraction mixture through Whatman No. 1 filter paper (GE Healthcare Life Sciences, Pittsburgh, PA, USA), and the solvent was removed using a rotary vacuum evaporator (Centra Evaporator; Bioneer, Daejon, Korea). Finally, the dried crude extracts were collected and used for further experiments, subsequently labeled fractions A-E (Fig. 1A). The total extract yields were calculated as the percentage weight of extract per $100 \mathrm{~g}$ S. miltiorrhiza root on a dry basis. These indicated that $S$. miltiorrhiza root extracts obtained using $30 \%$ ethanol gave the highest total extract yield (14.5\%), followed by $30 \%$ ethanol at $4^{\circ} \mathrm{C}(11.7 \%), 70 \%$ ethanol (8.2\%), $100 \%$ ethanol $(0.65 \%)$ and acetone $(0.58 \%)$.

Cell culture. Human gastric adenocarcinoma (AGS), prostate carcinoma (LNCaP), breast adenocarcinoma (MCF7), colorectal carcinoma (HCT116) and lung adenocarcinoma
(A549) cells were purchased from the American Type Culture Collection (Manassas, VA, USA). The AGS, LNCaP and A549 cells were cultured in RPMI-1640 medium with $10 \%$ FBS. HCT116 and MCF7 cells were cultured in DMEM with $10 \%$ FBS. In addition, the media contained penicillin $(100 \mathrm{U} / \mathrm{ml})$ and streptomycin $(100 \mu \mathrm{g} / \mathrm{ml})$. Cells were maintained in a humidified incubator with $5 \% \mathrm{CO}_{2}$ at $37^{\circ} \mathrm{C}$. Cells were subcultured every two days, and cells in the logarithmic growth phase were used for experiments.

Thin-layer chromatography (TLC) assay. TLC plates $(5 \times 10 \mathrm{~cm})$ were prepared by cutting the commercially available sheets (TLC Silica gel 60 F254; Merck, Darmstadt, Germany). The fractions extracted from $S$. miltiorrhiza were transferred and the plates were eluted in a closed chamber with a mobile phase consisting of methylene chloride, methanol and water at a ratio of 25:8:5, respectively. Pure tanshinone reagents, including cryptotanshinone, dihydrotanshinone I and tanshinone I were used as controls.

Cell cytotoxicity assay. Cells were seeded in 48-well plates at a concentration of $2 \times 10^{4}$ cells/well and incubated for $24 \mathrm{~h}$ at $37^{\circ} \mathrm{C}$ to allow the cells to adhere to the bottoms of the plates. Cell culture media were removed by aspiration, replaced with fresh media containing an extreme concentration $(250 \mu \mathrm{g} / \mathrm{ml})$ of each fraction, and incubated for $24 \mathrm{~h}$ in order to compare the cytotoxic effects of the fractions. Next, the cells were further incubated in the dark with MTT reagent $(0.5 \mathrm{mg} / \mathrm{ml})$ for $2 \mathrm{~h}$ at $37^{\circ} \mathrm{C}$. Subsequently, the MTT reagent-containing culture media was aspirated from each well, and DMSO was added to dissolve the formazan precipitate. The absorbance of each sample was measured using a Multiskan EX microplate reader (Thermo Fisher Scientific, Vantaa, Finland) at a wavelength of $540 \mathrm{~nm}$. Following analysis of the results to determine an appropriate concentration range, the assay was repeated using $0,5,10,20$ or $50 \mu \mathrm{g} / \mathrm{ml} \mathrm{S}$. miltiorrhiza extract or $0,1,5$ or $10 \mu \mathrm{M}$ tanshinone reagent

Observation of cellular morphology. Following the cell viability assay, cells treated with the three tanshinone reagents were examined for any morphological alterations. Cells were photographed using a Zeiss Axiovert 100 microscope (magnification, x400; Carl Zeiss Microscopy GmbH, Jena, Germany).

Statistical analysis. Results are expressed as the mean \pm standard error of the mean of three separate experiments. Statistical analyses were performed with GraphPad Prism 5 software for Windows (GraphPad Software, Inc., San Diego, CA, USA). The data were subjected to one-way analysis of variance, followed by Dunnett's multiple comparison tests. $\mathrm{P}<0.05$ was considered to indicate a statistically significant difference.

\section{Results}

Influence of solvent and extraction method on the cytotoxic activity of $S$. miltiorrhiza extracts. The composition and properties of the active constituents in an extract/fraction of plant material vary in response to extraction conditions, including the solvent, extraction time and temperature. Thus, 


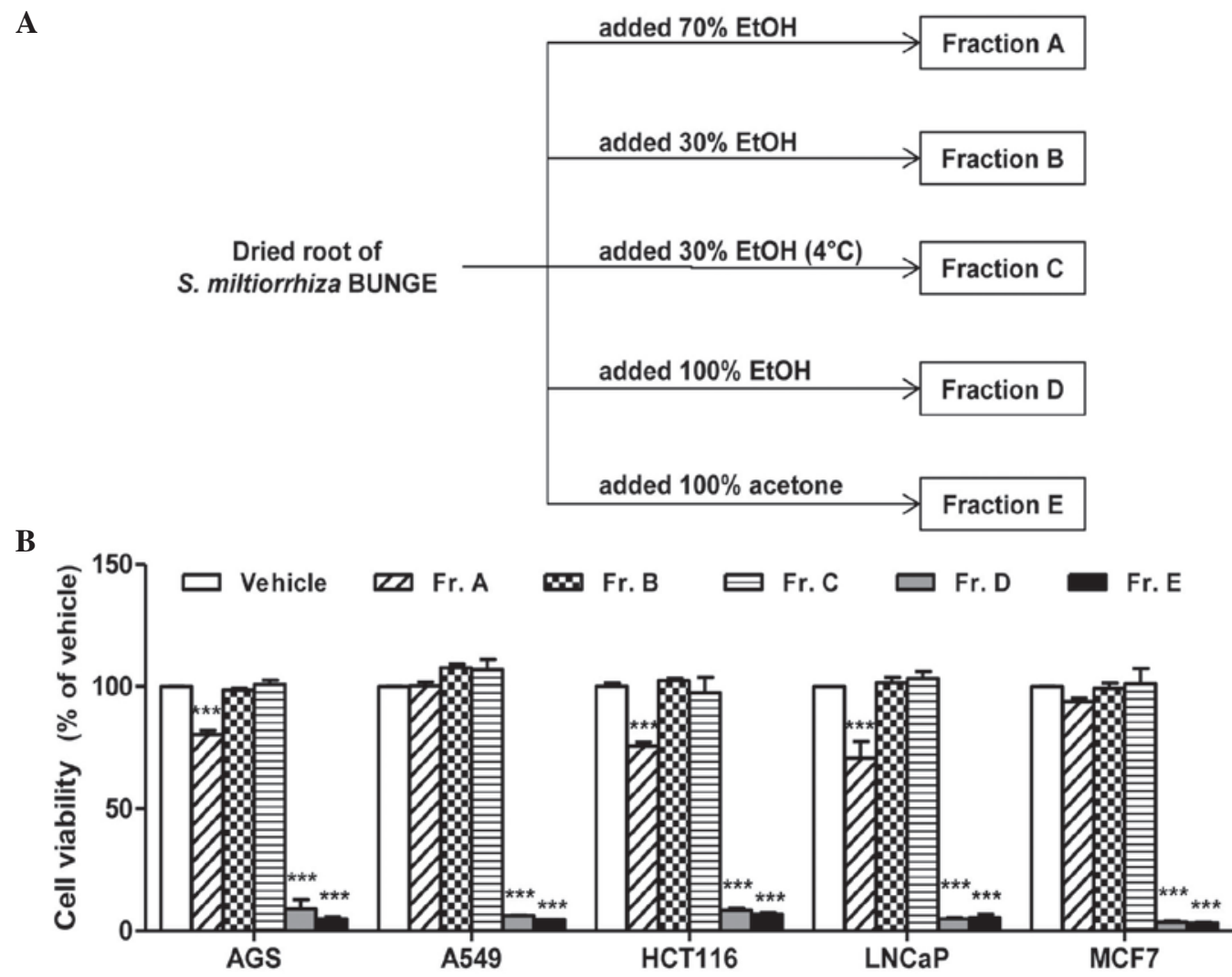

Figure 1. Effect of crude fractions on cell viability. (A) Scheme of crude fraction preparation. (B) Human cancer cells were treated with $250 \mu \mathrm{g} / \mathrm{ml}$ of fractions for $24 \mathrm{~h}$, and then cell viability was determined using the MTT assay. Results are expressed as the mean \pm standard error of the mean (n=3). ${ }^{* * *} \mathrm{P}<0.001 \mathrm{vs}$. vehicle-treated control. S. miltiorrhiza, Salvia miltiorrhiza; Fr., fraction; EtOH, ethanol.

the selection of the extraction method depends on the physical and chemical characteristics of the constituents being investigated. In the present study, cell viability was measured in the presence of an extreme concentration of each fraction $(250 \mu \mathrm{g} / \mathrm{ml})$, in order to compare the cytotoxic effects of the fractions. Fractions D (100\% ethanol) and E (100\% acetone) exhibited the most notable cytotoxic effect in all five cell lines compared with fractions A-C (Fig. 1B). Among the three fractions extracted with aqueous ethanol solvent (fractions A-C), only fraction A (70\% ethanol) produced a moderate reduction in cell viability; however, this effect was limited to the AGS, HCT116 and LNCaP cells. In addition fractions B and C, which were extracted using identical solvents but at different extraction temperatures, exhibited no significant differences in cytotoxicity.

Effect of ethanolic S. miltiorrhiza extracts on cell viability. Next, the concentration-dependent effects of the two most active fractions (D and E) on cancer cell viability were investigated. No differences were observed in the results of the MTT assays conducted using concentrations of fractions D and E ranging from 0 to $250 \mu \mathrm{g} / \mathrm{ml}$. Thus, $50 \mu \mathrm{g} / \mathrm{ml}$ was the highest concentration investigated for determining the cytotoxic effects of fractions D and $\mathrm{E}$ (data not shown). Cells were treated with various concentrations of fractions D or $\mathrm{E}$ and reductions of cell viability were identified using an MTT assay. All five tumor cell lines exhibited reductions in cell viability following a 24-h treatment with fractions D or E (Fig. 2). AGS and HCT116 cells were the most affected by the
S. miltiorrhiza extracts. The $\mathrm{IC}_{50}$ values (inhibitory concentration that reduces cell viability by $50 \%$ ) of fractions $\mathrm{D}$ and $\mathrm{E}$ in the HCT116 cells were 10.22 and $8.70 \mu \mathrm{g} / \mathrm{ml}$, respectively (Table I). Compared with the other four cell cancer cell lines, LNCaP cells exhibited lower sensitivity to the cytotoxic effects of fractions $\mathrm{D}$ and $\mathrm{E}$. In addition, although the $\mathrm{IC}_{50}$ values of fractions D and E in the MCF7 cells were comparable with those in LNCaP cells, a significant cytotoxic effect was only observed in MCF7 cells at concentrations of 20 and $50 \mu \mathrm{g} / \mathrm{ml}$ (Table I; Fig. 2).

Confirmation of the presence of the reference tanshinones in the active fractions of $S$. miltiorrhiza. Qiu et al previously reported that the ethanol extract of S. miltiorrhiza is rich in lipophilic constituents, including cryptotanshinone, tanshinone I and IIA, and dihydrotanshinone I (19). The present study investigated whether the ethanol- and acetone-extracted fractions of S. miltiorrhiza contained lipophilic tanshinones that were able to produce a reduction in cell viability. The presence of tanshinones in the fractions was initially confirmed by TLC assays, using purified dihydrotanshinone I, cryptotanshinone and tanshinone I as reference materials. Fractions D and E presented bands at similar positions (Fig. 3A). Furthermore, it was observed that the TLC bands of fractions D and E were similar to those of the reference tanshinones (structures presented in Fig. 3B). These results suggest that dihydrotanshinone I, cryptotanshinone and tanshinone I may be the primary constituents of the potent cytotoxic fractions of $S$. miltiorrhiza root. 
A

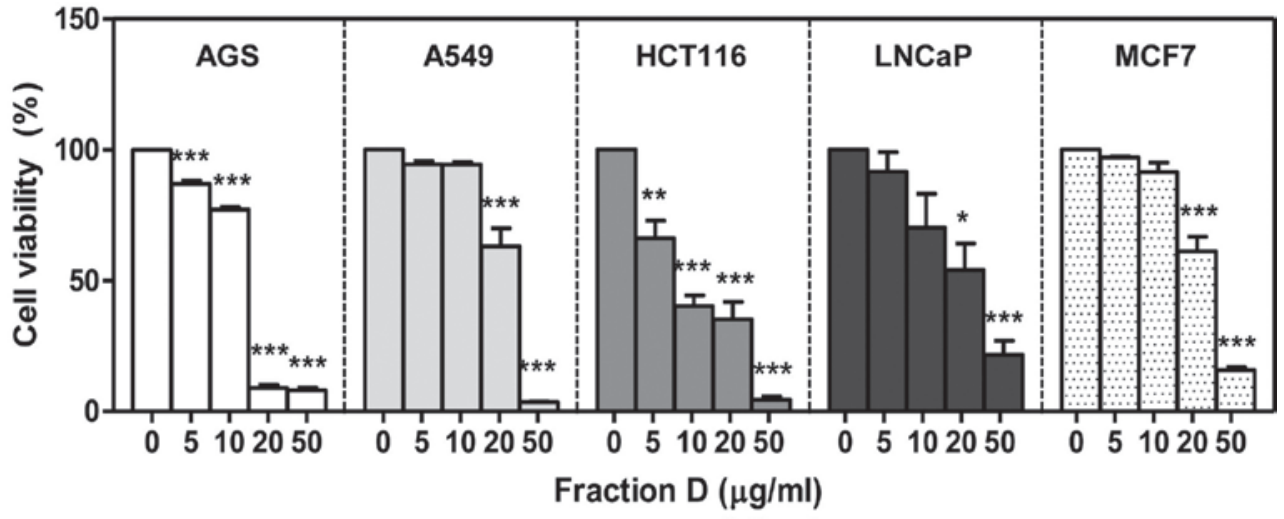

B

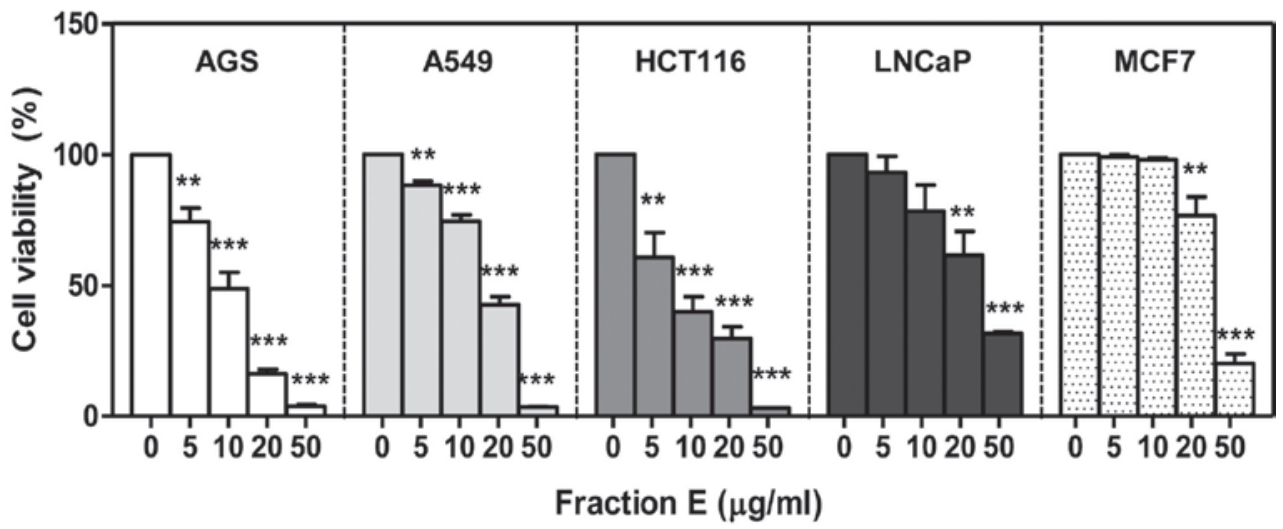

Figure 2. Effect of active fractions on cell viability. Human cancer cells were treated with various concentrations of fractions (A) D and (B) E for $24 \mathrm{~h}$, and cell viability was determined using an MTT assay. ${ }^{*} \mathrm{P}<0.05,{ }^{* *} \mathrm{P}<0.01$ and ${ }^{* * *} \mathrm{P}<0.001$ vs. vehicle-treated control.

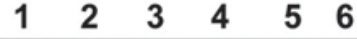

A

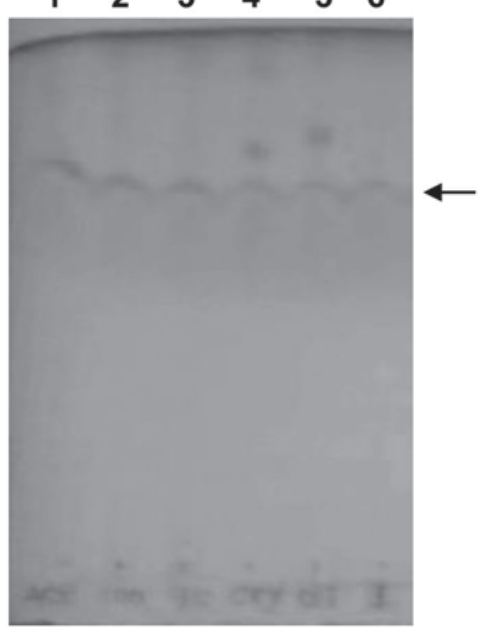

B<smiles>Cc1cccc2c1ccc1c3c4c(c(=O)c(=O)c=3c(=O)c12)C(C)C=C4</smiles>

Dihydrotanshinone I<smiles>CC1COC2=C1C(=O)C(=O)c1c2ccc2c1CCCC2(C)C</smiles>

Cryptotanshinone<smiles>Cc1coc2c1C(=O)C(=O)c1c-2ccc2c(C)cccc12</smiles>

Tanshinone I

Figure 3. Thin-layer chromatography (TLC) analysis of fractions and tanshinones from Salvia miltiorrhiza (Bunge). (A) TLC of fractions D and E of S. miltiorrhiza. Lane 1, fraction E; lanes 2 and 3, fraction D; lane 4, cryptotanshinone; lane 5, dihydrotanshinone I; and lane 6, tanshinone I. (B) Chemical structures of reference tanshinones.

Effects of reference tanshinones on the viability of human cancer cells. Next, it was determined whether the reference tanshinones alone were able to affect the viability of cancer cells. Cells were incubated with 1,5 or $10 \mu \mathrm{M}$ concentrations of the three tanshinones for $24 \mathrm{~h}$. All three of the examined tanshinones produced reductions in cell viability in a concentration-dependent manner; however, the extent of these effects varied (Fig. 4). Table II presents the $\mathrm{IC}_{50}$ values of dihydrotanshinone I, tanshinone I and crypto- tanshinone in various cancer cell lines. The $\mathrm{IC}_{50}$ values of the tanshinones in A549 cells were not determinable. In all five cancer cell lines, the $\mathrm{IC}_{50}$ value and cytotoxic potency of the three tanshinones descended in the following order: Dihydrotanshinone I $>$ tanshinone I $>$ cryptotanshinone (Table II). Among the three tanshinones investigated, dihydrotanshinone I reduced the cell viability with similar potency in the majority of cancer cell lines, with the exception of A549. 
Table I. Cytotoxic effect of extract fractions D and E on five human cancer cell lines $\left(\mathrm{IC}_{50}, \mu \mathrm{g} / \mathrm{ml}\right)$.

\begin{tabular}{lrrrrr}
\hline Fraction & AGS & A549 & HCT116 & LNCaP & MCF7 \\
\hline D & 10.44 & 15.72 & 10.22 & 22.65 & 22.15 \\
E & 9.22 & 13.64 & 8.70 & 29.86 & 25.50 \\
\hline
\end{tabular}

Table II. Cytotoxic effect of tanshinones from Salvia miltiorrhiza (Bunge) on five human cancer cell lines $\left(\mathrm{IC}_{50}, \mu \mathrm{M}\right)$.

\begin{tabular}{lccccc}
\hline Reagent & AGS & A549 & HCT116 & LNCaP & MCF7 \\
\hline DHTS & 3.35 & $>10$ & 3.87 & 5.29 & 8.02 \\
CTS & $>10$ & $>10$ & 8.84 & $>10$ & $>10$ \\
TS I & 8.21 & $>10$ & 5.82 & $>10$ & $>10$ \\
\hline
\end{tabular}

DHTS, dihydrotanshinone I; CTS, cryptotanshinone; TS I, tanshinone I.

Tanshinone-induced alteration of cellular morphology. The effects of the reference tanshinones on cellular morphology were observed directly using an optical microscope. Untreated HCT116 cells were homogeneously distributed on a cultured field, exhibiting a uniform polygonal shape (Fig. 5). Following incubation with the three tanshinones, various morphological changes were observed. Exposure of the cells to tanshinones transformed the shapes of the cells from polygonal to circular, and resulted in cell shrinkage. Furthermore, a reduction in cell number was observed and numerous floating cells were detected. Notably, these morphological alterations were observed at tanshinone concentrations as low as $1 \mu \mathrm{M}$. Similar modifications in morphology were observed in the AGS, LNCaP and MCF7 cells following incubation with the tanshinones (data not shown).

\section{Discussion}

The primary objective of the present study was to determine whether extracts of $S$. miltiorrhiza obtained with different solvents differ in their ability to induce cytotoxicity in five human cancer cell lines. Tanshinones, including cryptotanshinone, tanshinone I and IIA, and dihydrotanshinone I, have been reported to be the primary constituents of the ethanol extract of S. miltiorrhiza, and to exhibit the most marked cytotoxic effects (19). In addition, the present study investigated the possibility that these tanshinones were responsible for the cytotoxic activity of the ethanol extract of S. miltiorrhiza.

The results of the present study indicate that S. miltiorrhiza extract, obtained by extraction with $100 \%$ ethanol, effectively inhibits cancer cell growth. Solvent-based extraction methods may be advantageous compared with other methods of extracting active components from plant material due to their low cost and simplicity. Traditionally, numerous biologically active compounds have been extracted from plant materials using organic solvents, such as hexane, ether, acetonitrile, benzene and ethanol, at various dilutions $(20,21)$. However,
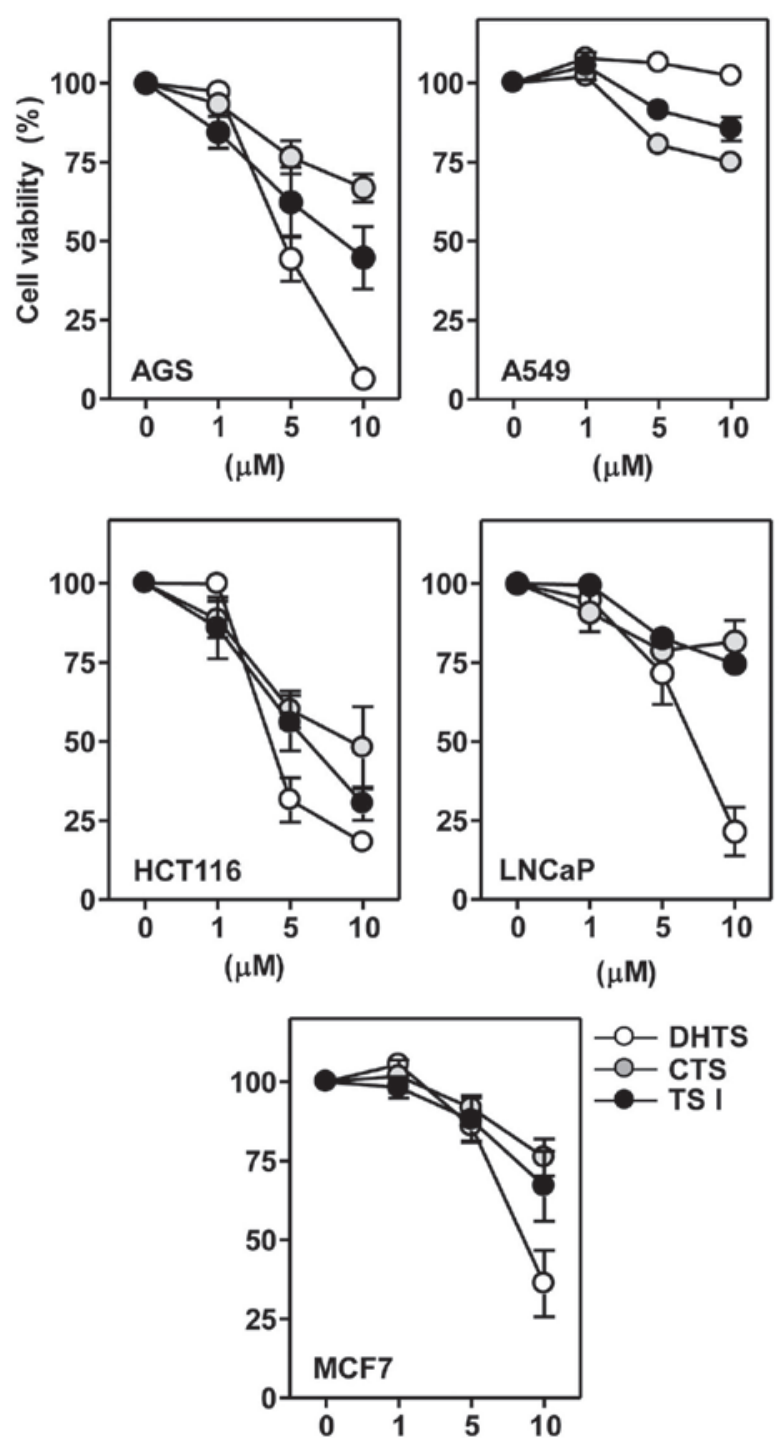

Figure 4. Cytotoxic effect of tanshinones on human cancer cell lines. The cells were treated with different concentrations of DHTS, CTS and TS I for $24 \mathrm{~h}$, and then percentage of cell survival was determined using the MTT assay. Results are expressed as the mean \pm standard error of the mean $(n=3)$. DHTS, dihydrotanshinone I; CTS, cryptotanshinone; TS I, tanshinone I.

these solvents may exert toxic effects in human patients, limiting their therapeutic potential (22). Thus, the solvent must be separable from the final extract, particularly if the product is intended to be used in food applications (21). Ethanol is a typical solvent used for plant extraction and is safe for human consumption. Ethanol is considered to be 'generally recognized as safe' by the US Food and Drug Administration designation for food additives, functional foods and dietary supplements (23). In the Republic of Korea particularly, ethanol is recommended for the preparation of food-grade extracts according to 'Regulation on approval of functional food ingredients for health functional food' (24). The results of the present study suggest that the ethanol extract of S. miltiorrhiza may be used as a functional food ingredient or as a dietary supplement for the enhancement of health.

In the present study, fraction D (100\% ethanol) exhibited the most potent cytotoxic activity, comparable to that of the acetone extract (fraction E; Fig. 1B). It is widely accepted 

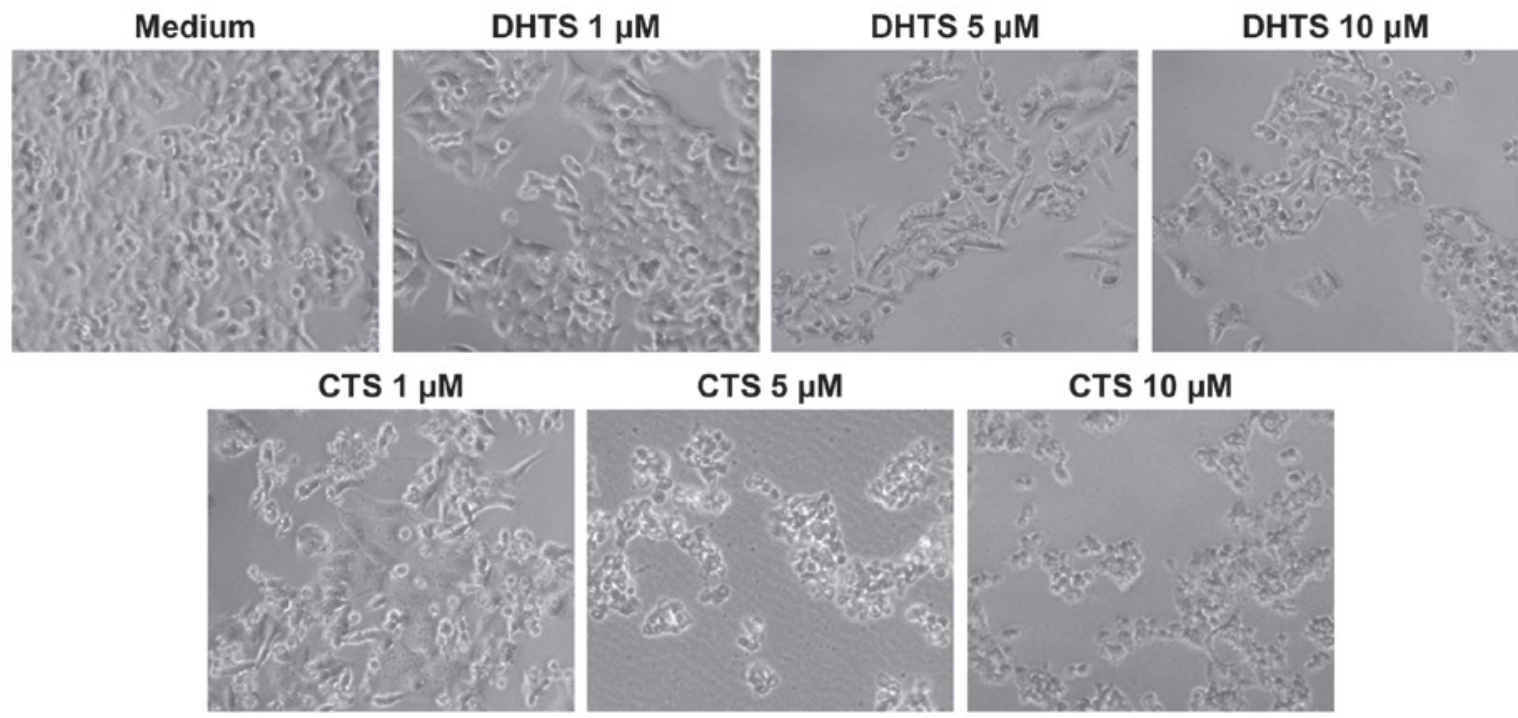

TS I $1 \mu \mathrm{M}$

TS I $5 \mu \mathrm{M}$

TS I $10 \mu \mathrm{M}$
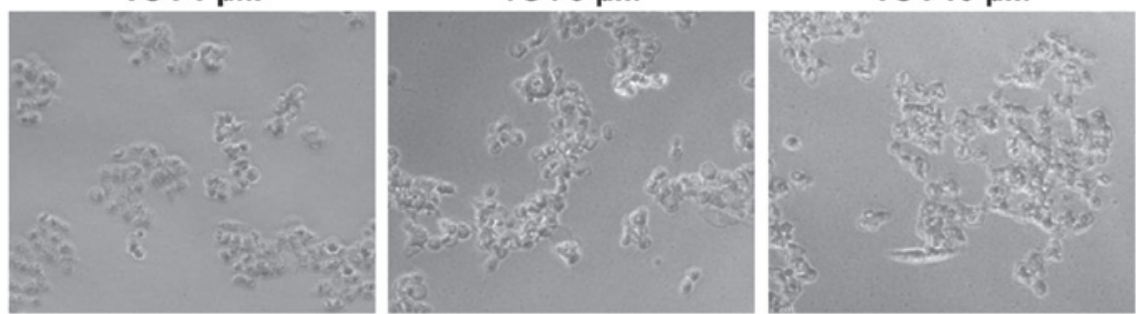

Figure 5. Effect of tanshinones on cell morphology. HCT116 cells were treated with different concentrations of DHTS, CTS and TS I for 24 h, and cell morphology was observed and photographed by phase contrast microscopy (magnification, x400). CTS, cryptotanshinone; DHTS, dihydrotanshinone I; TS I, tanshinone I.

that the specific components responsible for the anti-cancer activity of $S$. miltiorrhiza are diterpenoids with a furano-1,2or a furano-1,4-naphthoquinone skeleton, otherwise known as tanshinones. A previous study demonstrated that the ethanol extract of S. miltiorrhiza root contained $729.6 \mu \mathrm{g}$ dihydrotanshinone I, $352.4 \mu \mathrm{g}$ cryptotanshinone, $88.95 \mu \mathrm{g}$ tanshinone I and $649.9 \mu \mathrm{g}$ tanshinone IIA per gram of dried root (14). This may explain why fraction $\mathrm{D}$ produced the most notable reduction in the growth of cancer cells. However, further research is required in order to identify the chemical compositions of the individual $S$. miltiorrhiza extracts.

In the present study, differences in the efficacy of the extracts obtained using ethanol as a solvent at 30,70 and $100 \%$, suggested that the concentration of ethanol used affected the cytotoxic activity of the resulting extract. The $100 \%$ ethanol extract of S. miltiorrhiza exhibited the most marked cytotoxic effect, followed by 70 and $30 \%$. Previous studies have reported that the hydrophilic compounds present in S. miltiorrhiza exhibit anti-cancer activity in a number of tumor cell types (25-27). However, in the present study, the $30 \%$ ethanol extract (fraction B) was unable to induce toxic effects in any of the five cancer cell lines. It remains unclear why the $30 \%$ ethanol extract of S. miltiorrhiza was only minimally toxic to the cancer cells. The possibilities include the potency or selectivity of hydrophilic compounds in the extract. For example, protocatechualdehyde in the aqueous fraction of $S$. miltiorrhiza reduced cell growth of HCT116 cells by $21 \%$ at $50 \mu \mathrm{M}(28)$, while the $\mathrm{IC}_{50}$ values of the tanshinones in the present study were $<10 \mu \mathrm{M}$ in the same cell lines. The concentration of biologically active hydrophilic compounds in the $30 \%$ ethanol extract is another possible reason for the lack of observed cytotoxicity. Thus, there is a requirement for further studies to elucidate the chemical composition and concentrations of the active constituents of the ethanol extracts of S. miltiorrhiza.

To date, $\sim 1,700$ studies have been published on S. miltiorrhiza, $<200$ in association with cancer and more than half concerning tanshinones. Among the numerous compounds previously identified in S. miltiorrhiza, tanshinones have become increasingly studied due to their relatively high abundance and their ability to exert a cytotoxic effect by inhibiting growth and inducing apoptosis. In the present study, dihydrotanshinone I exhibited the most notable cytotoxicity against the human cancer cell lines, followed by tanshinone I and cryptotanshinone. These results are consistent with previous reports, which indicated that dihydrotanshinone I possesses the most marked cytotoxic effect among the three tanshinones examined in numerous human cancer cell lines $(14,29,30)$. As these three tanshinones possess similar chemical structures, the limited variations in these structures may be responsible for the differences in their cytotoxic effects. As presented in Fig. 3B, the only structural differences among the three reference tanshinones are in aromatic rings $\mathrm{A}$ and $\mathrm{C}$. A previous report indicated that the structure of ring A may contribute to the cytotoxic effect, resulting in the increased cytotoxic potential of dihydrotanshinone I and 
tanshinone I compared with cryptotanshinone, in terms of growth inhibition (29).

There are a limited number of studies regarding the effects of the active components of $S$. miltiorrhiza in humans. In China, S. miltiorrhiza is used extensively in various TCM preparations, and $\sim 80,000,000 \mathrm{~kg}$ of crude S. miltiorrhiza extract is consumed as a drug every year (31). The long historical use of this plant as a TCM indicates that S. miltiorrhiza produces limited side-effects and may be safe for human consumption. Studies of the anti-cancer potential of S. miltiorrhiza began in the early 1990 s, although it has been used for $>2,000$ years for medicinal purposes $(32,33)$. Studies published in the two subsequent decades have reported that $S$. miltiorrhiza extracts and tanshinones possess broad-range growth inhibitory activity against various cancer cell lines $(34,35)$. Furthermore, two reports have indicated that combinations of $S$. miltiorrhiza extracts with other medicinal plants modulate immunological functions in patients $(36,37)$. Capsules containing S. miltiorrhiza combined with the mushroom Trametes versicolor (known as yunzhi in China) appeared to alleviate lymphopenia to patients with nasopharyngeal carcinoma, when administered orally for 16 weeks during the course of radiotherapy (36). In patients with breast cancer, regular oral consumption of these capsules was observed to promote cell-mediated and humoral immunological functions, and thus exhibited an anti-cancer effect (37). No serious adverse side-effects were observed in these studies. Based on these previous studies, we hypothesize that $S$. miltiorrhiza may be used safely as an adjunct treatment. Thus, previous studies suggest that the consumption of the ethanol extract of $S$. miltiorrhiza as an ingredient in functional foods need not be limited to healthy individuals but may aid cancer patients and enhance the efficacy of standard cancer therapy.

In conclusion, the present study indicates that the ethanol extract of $S$. miltiorrhiza inhibits the growth of cancer cells. Furthermore, the tanshinone components in this extract appear to be capable of effectively reducing cancer cell viability. These results support the use of the ethanol extract of S. miltiorrhiza as a novel, efficacious and safe candidate for dietary supplements or as an ingredient in functional foods. However, future studies, involving clinically relevant animal models, are required to further elucidate the potential of S. miltiorrhiza extract as a therapeutic agent.

\section{Acknowledgements}

The present study was supported by the 2014 Post-Doc Development Program of Pusan National University (Busan, South Korea). The authors thank Aging Tissue Bank (Busan, South Korea) for providing research materials. The present study was also supported by the Research and Development Programme of the Ministry of Trade, Industry and Energy (MOTIE)/Korea Institute for Advancement of Technology (no. N0000697; Establishment of Infrastructure for Anti-Aging Industry Support) and the Research and Development Programme of the MOTIE/Korea Evaluation Institute of Industry Technology (no. 10040391; Development of Functional Food Materials and Devices for the Prevention of Aging-associated Muscle Function Decrease).

\section{References}

1. Cheng TO: Cardiovascular effects of Danshen. Int J Cardiol 121: 9-22, 2007.

2. Wasser S, Ho JM, Ang HK and Tan CE: Salvia miltiorrhiza reduces experimentally-induced hepatic fibrosis in rats. J Hepatol 29: 760-771, 1998.

3. Ahn YM, Kim SK, Lee SH, et al: Renoprotective effect of Tanshinone IIA, an active component of Salvia miltiorrhiza, on rats with chronic kidney disease. Phytother Res 24: 1886-1892, 2010.

4. Wen XD, Wang CZ, Yu C, et al: Salvia miltiorrhiza (dan shen) significantly ameliorates colon inflammation in dextran sulfate sodium induced colitis. Am J Chin Med 41: 1097-1108, 2013.

5. Li YG, Song L, Liu M, Hu ZB and Wang ZT: Advancement in analysis of Salviae miltiorrhizae Radix et Rhizoma (Danshen). J Chromatogr A 1216: 1941-1953, 2009.

6. Tao S, Zheng Y, Lau A, et al: Tanshinone I activates the Nrf2-dependent antioxidant response and protects against As(III)-induced lung inflammation in vitro and in vivo. Antioxid Redox Signal 19: 1647-1661, 2013.

7. Park EJ, Zhao YZ, Kim YC and Sohn DH: Preventive effects of a purified extract isolated from Salvia miltiorrhiza enriched with tanshinone I, tanshinone IIA and cryptotanshinone on hepatocyte injury in vitro and in vivo. Food Chem Toxicol 47: 2742-2748, 2009

8. Tao S, Justiniano R, Zhang DD and Wondrak GT: The Nrf2-inducers tanshinone I and dihydrotanshinone protect human skin cells and reconstructed human skin against solar simulated UV. Redox Biol 1: 532-541, 2013.

9. Mahesh R, Jung HW, Kim GW, Kim YS and Park YK: Cryptotanshinone from Salviae miltiorrhizae radix inhibits sodium-nitroprusside-induced apoptosis in neuro-2a cells. Phytother Res 26: 1211-1219, 2012.

10. Jeon SJ, Son KH, Kim YS, Choi YH and Kim HP: Inhibition of prostaglandin and nitric oxide production in lipopolysaccharide-treated RAW 264.7 cells by tanshinones from the roots of Salvia miltiorrhiza bunge. Arch Pharm Res 31: 758-763, 2008.

11. Trinh HT, Chae SJ, Joh EH, Son KH, Jeon SJ and Kim DH: Tanshinones isolated from the rhizome of Salvia miltiorrhiza inhibit passive cutaneous anaphylaxis reaction in mice. J Ethnopharmacol 132: 344-348, 2010.

12. Wang X, Morris-Natschke SL and Lee KH: New developments in the chemistry and biology of the bioactive constituents of Tanshen. Med Res Rev 27: 133-148, 2007.

13. Zhang Y, Jiang P, Ye M, Kim SH, Jiang C and Lu J: Tanshinones: sources, pharmacokinetics and anti-cancer activities. Int J Mol Sci 13: 13621-13666, 2012.

14. Lee WY, Chiu LC and Yeung JH: Cytotoxicity of major tanshinones isolated from Danshen (Salvia miltiorrhiza) on HepG2 cells in relation to glutathione perturbation. Food Chem Toxicol 46: 328-338, 2008.

15. Lee JH, Shin YJ, Kim HJ, Oh JH, Jang YP and Lee YJ: Danshen extract does not alter pharmacokinetics of docetaxel and clopidogrel, reflecting its negligible potential in P-glycoprotein- and cytochrome P4503A-mediated herb-drug interactions. Int J Pharm 410: 68-74, 2011.

16. Zhao Y, Hao Y, Ji H, et al: Combination effects of salvianolic acid B with low-dose celecoxib on inhibition of head and neck squamous cell carcinoma growth in vitro and in vivo. Cancer Prev Res (Phila) 3: 787-796, 2010.

17. Franek KJ, Zhou Z, Zhang WD and Chen WY: In vitro studies of baicalin alone or in combination with Salvia miltiorrhiza extract as a potential anti-cancer agent. Int J Oncol 26: 217-224, 2005.

18. Wang SX, Hunter $\mathrm{W}$ and Plant A: Isolation and purification of functional total RNA from woody branches and needles of Sitka and white spruce. Biotechniques 28: 292-296, 2000.

19. Qiu F, Jiang J, Ma Y, et al: Opposite effects of single-dose and multidose administration of the ethanol extract of Danshen on CYP3A in healthy volunteers. Evid Based Complement Alternat Med 2013: 730734, 2013.

20. Plaza M, Santoyo S, Jaime L, et al: Screening for bioactive compounds from algae. J Pharm Biomed Anal 51: 450-455, 2010.

21. Starmans DAJ and Nijhuis HH: Extraction of secondary metabolites from plant material: A review. Trends Food Sci Techol 7: 191-197, 1996.

22. Li BB, Smith B and Hossain MM: Extraction of phenolics from citrus peels I. Solvent extraction method. Sep Purif Technol 48: 182-188, 2006. 
23. United States Food and Drug Administration: Generally Recognized as Safe (GRAS). http://www.fda.gov/Food/ IngredientsPackagingLabeling/GRAS/. Accessed October 5, 2014.

24. Korean Food and Drug Administration: Regulation on approval of functional food ingredients for health functiona food. http://www.foodnara.go.kr/hfoodi/industry/main/sub jsp?Mode $=$ view \&boardID $=$ s_0502_bbs\&num $=96 \&$ tpage $=3 \& \mathrm{ke}$ yfield $=\& k e y=\& b C a t e=$. Accessed October 5, 2014.

25. Chang JY, Chang CY, Kuo CC, Chen LT, Wein YS and Kuo YH: Salvinal, a novel microtubule inhibitor isolated from Salvia miltiorrhizae Bunge (Danshen), with antimitotic activity in multidrug-sensitive and -resistant human tumor cells. Mol Pharmacol 65: 77-84, 2004

26. Bi L, Chen J, Yuan X, Jiang Z and Chen W: Salvianolic acid A positively regulates PTEN protein level and inhibits growth of A549 lung cancer cells. Biomed Rep 1: 213-217, 2013.

27. Yang Y, Ge PJ, Jiang L, Li FL and Zhu QY: Modulation of growth and angiogenic potential of oral squamous carcinoma cells in vitro using salvianolic acid B. BMC Complement Altern Med 11: 54, 2011.

28. Jeong JB and Lee SH: Protocatechualdehyde possesses anti-cancer activity through downregulating cyclin D1 and HDAC2 in human colorectal cancer cells. Biochem Biophys Res Commun 430: 381-386, 2013.

29. Li H, Zhang Q, Chu T, et al: Growth-inhibitory and apoptosis-inducing effects of tanshinones on hematological malignancy cells and their structure-activity relationship. Anticancer Drugs 23: 846-855, 2012.
30. Lee WY, Cheung CC, Liu KW, Fung KP, Wong J, Lai PB and Yeung JH: Cytotoxic effects of tanshinones from Salvia miltiorrhiza on doxorubicin-resistant human liver cancer cells. J Nat Prod 73: 854-859, 2010.

31. Wu W, Zhu Y, Zhang L, Yang R and Zhou Y: Extraction, preliminary structural characterization, and antioxidant activities of polysaccharides from Salvia miltiorrhiza Bunge. Carbohydr Polym 87: 1348-1353, 2012.

32. Zhou L, Zuo Z and Chow MS: Danshen: an overview of its chemistry, pharmacology, pharmacokinetics, and clinical use. J Clin Pharmacol 45: 1345-1359, 2005.

33. Wu WL, Chang WL and Chen CF: Cytotoxic activities of tanshinones against human carcinoma cell lines. Am J Chin Med 19: 207-216, 1991.

34. Gong Y, Li Y, Lu Y, et al: Bioactive tanshinones in Salvia miltiorrhiza inhibit the growth of prostate cancer cells in vitro and in mice. Int J Cancer 129: 1042-1052, 2011.

35. Liu L, Jia J, Zeng G, et al: Studies on immunoregulatory and anti-tumor activities of a polysaccharide from Salvia miltiorrhiza Bunge. Carbohydr Polym 92: 479-483, 2013.

36. Bao YX, Wong CK, Leung SF, et al: Clinical studies of immunomodulatory activities of Yunzhi-Danshen in patients with nasopharyngeal carcinoma. J Altern Complement Med 12: 771-776, 2006

37. Wong CK, Bao YX, Wong EL, Leung PC, Fung KP and Lam CW: Immunomodulatory activities of Yunzhi and Danshen in post-treatment breast cancer patients. Am J Chin Med 33: 381-395, 2005. 